\title{
Transtorno de estresse pós traumático sofridos por crianças abusadas sexualmente
}

\author{
Post traumatic stress disorder suffered by sexually abused children \\ Trastorno de estrés postraumático que sufren niños abusados sexualmente
}

Recebido: 02/10/2021 | Revisado: 08/11/2021 | Aceito: 10/11/2021 | Publicado: 12/11/2021

Grasiele dos Santos Negreiros de Araújo
ORCID: https://orcid.org/0000-0001-7979-6600
Centro Universitário Fametro, Brasil
E-mail: grasisnts @ gmail.com
Polliana Gato Martins
ORCID: https://orcid.org/0000-0001-8688-4641
Centro Universitário Fametro, Brasil
E-mail: polliana.gato51 @ gmail.com

\begin{abstract}
Resumo
Os transtornos psicológicos sofridos por crianças abusadas sexualmente, mas especificamente o Transtorno de Estresse Pós Traumático-TEPT. A pesquisa tem como objetivo analisar O Transtorno de Estresse Pós Traumático sofridos Por Crianças Abusadas Sexualmente. Esta pesquisa terá uma abordagem qualitativa de caráter bibliográfico, tendo como instrumento de pesquisa livros didáticos a respeito do tema, portais eletrônicos de periódicos científicos entre 2008 a 2021. Nesta etapa, foram utilizados 6 artigos, 15 trabalhos publicados em revistas e 3 livros. O atendimento psicológico oferece recursos para a compreensão no processo de adoecimento e estratégias de enfrentamento, neste caso é de suma importância o atendimento psicológico após sofrer abusos sexuais, para que o sujeito possa expor o que está sentindo e o profissional consiga buscar técnicas para recuperar o que foi lhe tirado. Sobre os métodos terapêuticos é pouco encontrado, bem como a ausência de instrumentos psicológicos validados e eficazes para avaliação clínica das crianças e dos adolescentes. Observou-se que o transtorno de estresse póstraumático é o mais comum em crianças vítimas de abuso sexual e que a Teoria Cognitivo-comportamental é o modelo a ser desenvolvido e aplicado nas mesmas.
\end{abstract}

Palavras-chave: Abuso sexual; Crianças; TEPT.

\begin{abstract}
Psychological disorders suffered by sexually abused children, but specifically Post Traumatic Stress Disorder-PTSD. The research aims to analyze Post Traumatic Stress Disorder suffered by Sexually Abused Children. This research will have a qualitative approach of bibliographic character, having as research instrument textbooks on the subject, electronic portals of scientific journals between 2008 and 2021. In this step, 6 articles, 15 works published in journals and 3 books were used. Psychological care offers resources for understanding the disease process and coping strategies. In this case, psychological care after suffering sexual abuse is of paramount importance, so that the subject can expose what he is feeling and the professional can seek techniques to recover the that was taken from him. There is little information about therapeutic methods, as well as the absence of validated and effective psychological instruments for the clinical assessment of children and adolescents. It was observed that post-traumatic stress disorder is the most common in children victims of sexual abuse and that the Cognitive-Behavioral Theory is the model to be developed and applied to them.
\end{abstract}

Keywords: Sexual abuse; Children; PTSD.

\section{Resumen}

Trastornos psicológicos sufridos por niños abusados sexualmente, pero específicamente Trastorno de estrés postraumático-TEPT. La investigación tiene como objetivo analizar el trastorno de estrés postraumático que sufren los niños abusados sexualmente. Esta investigación tendrá un enfoque cualitativo de carácter bibliográfico, teniendo como instrumento de investigación libros de texto sobre el tema, portales electrónicos de revistas científicas entre 2008 y 2021. En este paso se utilizaron 6 artículos, 15 trabajos publicados en revistas y 3 libros. La atención psicológica ofrece recursos para la comprensión del proceso de la enfermedad y estrategias de afrontamiento. En este caso, la atención psicológica después de sufrir un abuso sexual es de suma importancia, para que el sujeto pueda exponer lo que está sintiendo y el profesional pueda buscar técnicas para recuperar lo que fue tomado. de él. Existe poca información sobre los métodos terapéuticos, así como la ausencia de instrumentos psicológicos validados y efectivos para la evaluación clínica de niños y adolescentes. Se observó que el trastorno por estrés postraumático es el más común en niños víctimas de abuso sexual y que la Teoría Cognitivo-Conductual es el modelo a desarrollar y aplicarles.

Palabras clave: Abuso sexual; Niños; TEPT. 


\section{Introdução}

O abuso sexual praticado contra crianças é um dos grandes problemas da sociedade brasileira, também é um problema de saúde pública devido as incidências e graves consequências para o desenvolvimento cognitivo, bem como, afetivo e social da vítima e de sua família (Kowalczyk, 2019).

O abuso sexual também pode ocorrer em diferentes situações, muitas vezes fora do espaço familiar, ou seja, o abuso sexual pode ocorrer em situações nas quais crianças e adolescentes são envolvidos em pornografia e exploração sexual (Wang, Tang \& Liu, 2020).

Ribeiro et al. (2018) destaca que “as vítimas possuem inúmeros danos no que tange, principalmente, as relações afetivas. Há uma intensa dificuldade para que elas consigam construir vínculos de confiança”. Ainda conforme os autores, "percebe-se também que estas desenvolvem baixa autoestima, gerando bloqueios relacionados a insegurança e posteriormente vindo a se associar com transtornos como ansiedade e depressão".

A exposição ao trauma e, por muitas vezes, o fato da criança se calar, podem ocasionar vários transtornos que ocasionam sofrimento psicológico cotidiano intenso, tornando difícil a tarefa de desempenhar ações que, até determinado momento de suas vidas, eram consideradas simples (Russell, Higgins \& Posso, 2020). Dentre os principais transtornos apresentados por crianças vítimas de abuso sexual, tem-se o transtorno de ansiedade, o transtorno de adaptação e o Transtorno de Estresse Pós-Traumático - TEPT, objeto de estudo desse artigo (Hébert, Langevin \& Oussaïd, 2018).

Para Letourneau et al. (2018), o interesse de se pesquisar sobre os transtornos psicológicos causados pelo abuso sexual de crianças deve-se ao fato dos inúmeros casos relatados nas últimas décadas, visto que os abusos sexuais tiveram um crescimento elevado no Brasil e no mundo.

Esta pesquisa contribui com a discussão ao disponibilizar novas abordagens e análises sobre o abuso sexual infantil, transtornos psicológicos causados por quem sofre abusos sexuais, Transtorno de Estresse Pós Traumático-TEPT em crianças também como esse se dará o desenvolvimento na vida adulta.

A relevância desta pesquisa é a possibilidade sobre o transtorno de estresse pós traumático que pode ser abordado em conjunto com o tratamento em psicoterapia também será uma troca de informações a respeito dos temas acima mencionados.

No que se refere à contribuição para a sociedade os resultados desta pesquisa apresentarão diversas maneiras como a criança e adolescente pode levar sua vida adiante, mostrando como o tratamento adequado pode beneficiar sua vida adulta, possibilitando uma vida psíquica saudável sem maiores prejuízos no futuro.

Desta forma o presente estudo tem o objetivo de analisar o TEPT sofridos por crianças abusadas sexualmente. Além disso os objetivos específicos são: apresentar os tipos de transtornos psiquiátricos; mostrar o que é abuso sexual praticado contra a criança; relatar os sinais apresentados por crianças vítimas de violência sexual no contexto do TEPT; discutir de que forma os tratamentos adequados colabora no TEPT.

\section{Metodologia}

O processo metodológico tem como propósito nortear o pesquisador no caminho que será percorrido durante tal pesquisa, na qual investigará a relação da teoria e prática. Procedimento é o modo como algo é feito, através dele pode ser descrito o tipo de pesquisa e instrumentos que serão utilizados para a coleta e a interpretação dos dados que ajudarão na solução do problema.

A pesquisa bibliográfica segundo Soares et al. (2018, p.11) ela "procura explicar e discutir um assunto, tema ou problema com base em referências publicadas em livros, periódicos, revistas, enciclopédias, dicionários, jornais, sites, CDs, anais de congressos etc". A indicação para esses estudos relaciona-se ao fato de a aproximação com o objeto ser dada a partir 
de fontes bibliográficas. Os instrumentos que serão utilizados nesta pesquisa bibliográfica serão livros didáticos, portais eletrônicos como Scielo, capes, revistas cientificas e google academico.

Trata-se de uma pesquisa qualitativa que segundo Flick (2008) é um processo contínuo de construção que mostra a realidade através do estudo, como: Discursos ou falas dos sujeitos da pesquisa sobre os fatos evidenciados.

Em seguida o material apurado viabilizou a identificação do projeto de pesquisa sobre abuso sexual e os transtornos sofridos por crianças abusadas sexualmente.

Neste estudo, essa classificação foi realizada por meio da criação de uma tabela para agrupar os principais procedimentos de coleta. A coleta de dados foi feita através de busca de artigos e livros utilizando os termos: abuso sexual, crianças, TEPT, tratamento psicoterápico e saúde mental, na plataforma digitai Scielo.

$\mathrm{Na}$ análise de dados há um rigor científico para que os mesmos sejam organizados de forma que as informações ali expostas sejam claras e objetivas. Para a realização da análise, conforme delineamento da metodologia, foi feito uma análise descritiva conforme a metodologia proposta e baseada no referencial teórico construído para a pesquisa a partir de coletas de informações de fontes primárias, através de revisão sistemática de dados e análise de conteúdo dos manifestos apresentados.

Do ponto de vista legal, cita-se a lei 9.610/98, artigo $25^{\circ}$ no qual "é vedada ao acadêmico a citação de obras sem a respectiva indicação do autor", se caracterizando como plágio.

Foram considerados critérios de inclusão de pesquisa artigos de revistas científicas indexadas, referentes a pesquisas empíricas, publicadas nas bases de dados Scielo, Capes, e livros técnicos dos anos de 2008 a 2021 . Foram considerados critérios de exclusão artigos de revistas cientificas e livros que não sejam do ano de 2008 a 2020. Nesta etapa, foram utilizados 8 artigos, 15 trabalhos publicados em revistas e 3 livros.

\section{Resultados}

\subsection{Os tipos de transtornos psiquiátricos}

Não é fácil se falar sobre os transtornos psiquiátricos. Ao longo do tempo, eles se apresentaram de forma controversa, em relação a sua simbologia, inserção cultural e teorias. Atualmente, ainda é visto como um tabu e como uma não-aceitação daqueles que possuem algum tipo de transtorno ou que convivem com pessoas que apresentam os mesmos (Sibrava, Bjornsson \& Pérez Benítez, 2019).

Na pesquisa de Clark (2018), percebe-se que até o período do renascimento, o único transtorno tratado era a loucura. Após perpassar por várias definições e diversos métodos de tratamento, foi, no século XVII que a loucura passa a ser vista como uma desadaptação à sociedade e as internações em espaços físicos hospitalares torna-se mais frequente. No século XVIII, ocorre a reforma dos manicômios, com o objetivo de estabelecer higiene pública e reduzir riscos de contaminação (Equestre, 2019).

No Brasil, segundo os estudos de Meyer (2021), o primeiro hospital psiquiátrico foi criado em 1852, com o nome de Hospital Dom Pedro II, mas que ficou conhecido como "palácio dos loucos". Esse hospício tinha como princípio o isolamento da família e da sociedade pois, acreditava-se que esses fatores intensificariam a alienação mental (Facchinetti \& Jacó-Vilela, 2019).

A lei $\mathrm{n}^{\circ} 10.216$, de 6 de abril de 2001, dispõe sobre a proteção e os direitos das pessoas portadoras de transtornos mentais e redireciona o modelo assistencial em saúde mental. Em seu artigo $6^{\circ}$, afirma que a internação psiquiátrica somente será realizada mediante laudo médico circunstanciado que caracterize os seus motivos. Conforme o parágrafo único desse artigo, os tipos de internações são: internação voluntária: aquela que se dá com o consentimento do usuário; internação involuntária: aquela que se dá sem o consentimento do usuário e a pedido de terceiro e a internação compulsória determinada pela justiça. 
Conforme Schlag et al. (2021), o transtorno psiquiátrico é caracterizado por alterações cognitivas e comportamentais que prejudicam o indivíduo no seu cotidiano, nas suas relações sociais, pessoais e no convívio familiar e que causa sofrimento não somente para o indivíduo mais também para seus familiares. Ressalta-se que comportamentos anormais persistentes e recorrentes que causam danos é que podem ser considerados como transtorno (Sullivan \& Geschwind, 2019).

Dentre as principais doenças mentais, conforme a Associação Americana de Psiquiatria (2013), são: esquizofrenia, transtorno afetivo bipolar, psicose, transtorno de ansiedade, transtorno de personalidade, transtorno obsessivo compulsivo, transtorno de estresse, transtornos de ansiedade, transtornos depressivos, dentre outros.

Na pesquisa de Williams \& Beidas (2019), destaca-se que todos esses distúrbios carecem de compreensão, atenção e tratamento. Faz-se necessário desmitificar o "louco" e oferecer apoio médico, familiar e social para que o indivíduo, detentor de direitos, possa reconstruir seu psicológico e lidar com as adversidades do mundo (Kaufman et. al, 2019).

\subsection{Abuso sexual infantil}

A infância é a principal etapa do desenvolvimento do ser humano. Até a idade média, a criança era vista e submetida a vida adulta. Não existia um olhar sobre a infância. Existia uma diferenciação entre as crianças conforme sua classe social através das vestimentas. Conforme Ghandour et. al (2019), os adultos não se importavam com as crianças devido as más condições sanitárias, pois acreditavam que elas facilmente contrairiam alguma doença e morreriam.

De acordo com Keddie (2018), foi no século XV que a infância passou a ser diferenciada da vida adulta. Surgiram mais afetividades entre as famílias e uma relevante preocupação com a educação das crianças. Partindo disso, escolas foram criadas para que essas colocassem regras e ordem nas crianças, disciplinando-as. Segundo Kaneko (2021), a escola se encarregava da educação escrita e da etiqueta e a igreja da educação moral e ética.

Com o passar do tempo, a infância e a concepção de criança passaram por várias construções, conforme seu tempo e seu espaço, sendo distinguida pelo meio social ao qual eram inseridas. Atualmente, a criança é concebida como um ser em constante transformação e merece uma educação de qualidade, visto que ela tem seus direitos e deveres prescritos em leis (Garlen et. al, 2020).

O fenômeno de violência contra crianças é algo construído de forma social e historicamente, sendo os primeiros casos relatados historicamente no período da Revolução Industrial, aonde as crianças trabalhavam e eram vítimas de violência sexual. Segundo Skelton (2019), tal fenômeno encontra suas raízes na vida em sociedade e não no desenvolvimento biológico humano.

Segundo Ward et al (2018), existem quatro tipos de violência contra a criança: abuso físico, abuso psicológico ou emocional, abuso sexual e negligência. Ressalta-se que no contexto da violência sexual infantil existe diferença entre exploração sexual e violência sexual, pois a violência se configura como a estimulação sexual da criança com fins de satisfação do agressor e pode ocorrer dentro ou fora da família, enquanto que a exploração tem nas práticas sexuais com crianças uma forma de comércio e geralmente ocorre fora da família.

O abuso sexual contra crianças e adolescentes é considerado um grave problema de saúde pública. Conforme Debowska et al (2019), é problema de saúde pública devido aos altos índices de incidência e às sérias consequências para o desenvolvimento cognitivo, afetivo e social da vítima e de sua família. Destaca-se que o abuso sexual é um fenômeno transgeracional, que perpassa todas as classes sociais, mas que, nem sempre, é levado as autoridades competentes.

Pereda \& Díaz-Faes (2020), afirmam que a violência sexual é caracterizada por atos praticados com finalidade sexual que, por serem lesivos ao corpo e a mente do sujeito violado (crianças e adolescentes). Tal ato desrespeita aos direitos e as garantias individuais como liberdade, respeito e dignidade previstos na Lei no 8.069/90 - Estatuto da Criança e do Adolescente - ECA (BRASIL, 1990). 
Segundo Brown et al (2019), além de um grave problema de saúde pública, o abuso/violência sexual constitui um crime violento, reconhecido como desafio social e gera diversos problemas envolvendo consequências físicas, emocionais, podendo desenvolver transtornos (como estresse pós-traumático) e diversos comprometimentos da esfera psicossocial, comportamental e cognitiva, sem falar nos preconceitos.

O abuso e a violência sexual é um problema que atinge a vários países no mundo. Em relação ao Brasil, Platt et al (2018), descrevem que a violência sexual contra crianças e adolescentes ocupa segundo maior tipo de violência, perdendo somente para a violência física. A síndrome do segredo normalmente ocorre nas famílias com dinâmica de abuso, favorecendo sua continuidade. Afirma-se que a maioria das mães se calam por medo de perder seu cônjuge ou de ser agredida pelo mesmo. A criança se cala por acreditar que ninguém dará credibilidade ao que a mesma diz (da Silva \& Roncalli, 2018).

Conforme Monteiro et al. (2019), o abuso sexual constitui uma experiência traumática, resultando em prejuízos que podem se prolongar até a vida adulta, representando um fator de risco para o desencadeamento de diversas patologias e alterações de diversas ordens (psicológicas e funcionais) como depressão, pensamentos e comportamento suicida, ansiedade, estresse pós-traumático, entre outros.

Nascimento, Santana \& Marques (2021), indicam que somente após a criação do Estatuto da Criança e do Adolescente (ECA) em 1990 e do início das campanhas contra o abuso sexual foi que a questão do abuso e da violência sexual ganharam maior visibilidade do Brasil, mas ressalta também que ainda falta uma maior sensibilização da sociedade em relação ao tema.

\subsection{O TEPT e os sinais apresentados por crianças vítimas de violência sexual}

A violência contra crianças e adolescentes é considerada um grave problema de saúde pública pois, ocorre com frequência e ocasiona graves consequências para o desenvolvimento infantil. Dentre os tipos de violência, tem-se o abuso sexual, o abuso físico, o abuso psicológico, o abandono e a negligencia (ECA, 1990).

Segundo Walker-Descartes et al. (2021), o dano psicológico pode estar relacionado aos seguintes itens: a idade do início do abuso; a duração do mesmo; o grau de violência ou ameaça; a diferença de idade entre quem comete o abuso e a vítima; quão estreitamente era a relação da pessoa que cometeu o abuso com a pessoa que sofreu o abuso; a ausência de figuras parentais protetoras e o grau de segredo. Elmore \& Crouch (2020) afirmam que crianças vítimas de violência sexual tendem a desenvolverem transtorno de ansiedade, sintomas depressivos e agressivos, transtorno afetivo, transtorno de estresse póstraumático, distúrbios alimentares, dependência química e transtornos psicossexuais.

O TEPT é caracterizado pelo desenvolvimento e sintomas característicos após a exposição ao trauma como ameaça de morte, lesão grave ou violência sexual em uma das seguintes formas: vivência direta, testemunhar pessoalmente o evento traumático com outras pessoas, saber que o evento traumático ocorreu com alguém próximo ou ser exposto de forma repetida a detalhes do trauma (APA, 2013).

A Associação Psiquiátrica Americana APA (2013), define que para o diagnóstico de TEPT para adultos, adolescentes e crianças maiores de seis anos, faz-se necessário a exposição a um episódio concreto de ameaça de morte, violência sexual ou lesão grave de forma direta, indireta ou de forma repetida como critério principal.

Para a APA (2013), são critérios:

A. Exposição a episódio concreto ou ameaça de morte, lesão grave ou violência sexual. Vivenciar diretamente o evento traumático. Testemunhar pessoalmente o evento traumático ocorrido com outras pessoas. Saber que o evento traumático ocorreu com familiar ou amigo próximo. Nos casos de episódio concreto ou ameaça de morte envolvendo um familiar ou amigo, é preciso que o evento tenha sido violento ou acidental. 
B. Presença de um (ou mais) dos seguintes sintomas intrusivos associados ao evento traumático, começando depois de sua ocorrência. Lembranças intrusivas angustiantes, recorrentes e involuntárias do evento traumático. 2.Sonhos angustiantes recorrentes nos quais o conteúdo e/ou o sentimento do sonho estão relacionados ao evento traumático.

C. Evitação persistente de estímulos associados ao evento traumático, começando após a ocorrência do evento. Evitação ou esforços em recordações, pensamentos ou sentimentos angustiantes acerca de ou associados de perto ao evento traumático.

D. Alterações negativas em cognições e no humor associadas ao evento traumático começando ou piorando depois da ocorrência de tal evento. Sentimentos de distanciamento e alienação em relação aos outros.

E. Alterações marcantes na excitação e na reatividade associadas ao evento traumático, começando ou piorando após o evento, conforme evidenciado por dois (ou mais) dos seguintes aspectos: Problemas de concentração. Perturbação do sono.

F. A perturbação (Critérios B, C, D e E) dura mais de um mês.

G. A perturbação causa sofrimento clinicamente significativo e prejuízo social, profissional ou em outras áreas importantes da vida do indivíduo.

H. A perturbação não se deve aos efeitos fisiológicos de uma substância (p. ex., medicamento, álcool) ou a outra condição médica.

O transtorno pode ser especialmente grave ou duradouro quando o estressor é interpessoal e intencional (p. ex., tortura, violência sexual). O evento traumático pode ser revivenciado de diversas maneiras. É comum que a pessoa tenha lembranças recorrentes, involuntárias e intrusivas do evento. O TEPT é com frequência caracterizado por hipersensibilidade a ameaças potenciais, incluindo as relacionadas à experiência traumática.

Assim, existem diversos tratamentos psíquicos para crianças vitimizadas sexualmente, sendo que a terapia cognitivocomportamental (TCC) é considerada por Kizilhan, Steger \& Noll-Hussong (2020), como superior na intervenção com pacientes expostos a violência sexual e no manejo de sintomas provenientes do TEPT, por apresentar resultados significativos em comparação a outras abordagens.

\section{Discussão}

Nos estudos de Paul (2019), o TEPT caracteriza-se pelo quadro clínico que surge após a exposição ao evento traumático, o qual desencadeia sintomas de medo intenso, horror ou impotência. Ainda conforme o autor, a criança também pode manifestar comportamento agitado com sonhos aflitivos.

Os traumas provenientes do abuso sexual contra crianças ocasionam um grande impacto negativo em seu desenvolvimento, afetando as áreas cognitivas, comportamentais e social de uma criança. A saúde mental dos pais, conflito conjugal, dinâmica familiar, presença de eventos estressores, o nível socioeconômico da família, fatores culturais e comunitários influenciam no grau e manutenção da melhoria do TEPT (Miragoli, Camisasca \& Di Blasio, 2017).

Segundo Bassitt (2012), o TEPT causa alterações cognitivas e comportamentais que prejudicam o indivíduo no seu cotidiano, nas suas relações sociais, pessoais e no convívio familiar e que causa sofrimento não somente para o indivíduo mais também para seus familiares. Ressalta-se que comportamentos anormais persistentes e recorrentes que causam danos é que podem ser considerados como transtorno que precisa ser tratado.

Soares; Santos; Donadon (2021, p.7), “observou-se que, associadas ao TEPT, há mudanças estruturais em áreas cerebrais, como a alteração do volume da amígdala, do hipo-campo e do corpo caloso". Sinalizam ainda, também 
hiperatividade da amígdala, o que explica a alta responsividade e a sensação de ameaça vivenciada por crianças acometidos pelo TEPT. Desta maneira as crianças sofrem consequências traumáticas que poderá influenciar em sua fase adulta.

Soares; Santos; Donadon (2021, p.3) falam sobre "o tratamento medicamentoso pode ser favorável para o alívio de alguns sintomas, como agitação, ansiedade, insônia e depressão". Contudo os métodos terapêuticos podem contribuir para diminuir os efeitos traumáticos em crianças com TEPT, vítima de violência sexual.

Os autores citados discutem que os estudos sobre avaliação de métodos terapêuticos é pouco encontrado e verifica-se um outro problema que é a ausência de instrumentos psicológicos validados e eficazes para avaliação clínica das vítimas. Os autores discorrem sobre o TEPT é o mais comum em crianças vítimas de abuso sexual e que a Teoria Cognitivocomportamental é o modelo a ser desenvolvido e aplicado nas mesmas pois apresenta alta eficácia na redução de sintomas.

Deve-se levar em consideração que para a vítima, falar sobre a violência sexual é doloroso e se deve ter um olhar cauteloso, cuidadoso e uma escuta qualificada. É necessário que haja um acompanhamento multidisciplinar e que toda a família seja envolvida, não apenas a vítima. A qualidade dessa equipe ao abordar o tema é o primeiro passo para a intervenção.

\section{Considerações Finais}

No que se refere o TEPT é caracterizado pelo desenvolvimento e sintomas característicos após a exposição ao trauma como ameaça de morte, lesão grave ou violência sexual, onde se destaca a vivência direta, testemunhar pessoalmente uma situação traumática com outras pessoas, saber que o evento traumático ocorreu com alguém próximo ou ser exposto de forma repetida a detalhes do trauma. Assim, observou-se que esta exposição pode trazer grandes prejuízos psicológicos para a criança e os adolescentes na sua vida adulta, bem como desenvolver certos traumas como o TEPT.

Os traumas provenientes do abuso sexual contra crianças e adolescentes podem ocasionar um grande impacto negativo em seu desenvolvimento, afetando as áreas cognitivas, comportamentais e social. Para realizar um tratamento com esta criança e estes adolescentes com traumas psicológicos é necessário um acompanhamento de vários profissionais, bem como da família de forma integral.

Sobre os métodos terapêuticos é pouco encontrado, bem como a ausência de instrumentos psicológicos validados e eficazes para avaliação clínica das crianças e dos adolescentes. Observou-se que o TEPT é o mais comum em crianças vítimas de abuso sexual e que a Teoria Cognitivo-comportamental é o modelo a ser desenvolvido e aplicado nas mesmas, pois apresenta alta eficácia na redução de sintomas e traumas causadas pela violência sexual.

Percebeu-se que o tratamento para o TEPT precisa ser adequado e no tempo correto, desta maneira pode ajudar essas crianças a voltar a ter uma vida saudável e sem danos psicológicos na vida adulta. $\mathrm{O}$ atendimento psicológico oferece recursos para a compreensão no processo de adoecimento e estratégias de enfrentamento, neste caso é de suma importância o atendimento psicológico após sofrer abusos sexuais, para que o sujeito possa expor o que está sentindo e o profissional consigo buscar técnicas para recuperar o que foi lhe tirado. Desta forma, este estudo é apenas um esboço, que poderá servir e contribuir para novas pesquisas voltada para este contexto.

Desta maneira, este estudo é apenas um esboço que poderá contribuir para novas pesquisas voltadas para esta temática, podendo ser incluído novos autores que discutem o assunto, bem como, novos métodos terapêuticos que contribuam para o Tratamento do TEPT.

\section{Referências}

APA. (2013). Manual diagnóstico e estatístico de transtornos mentais. Porto Alegre: Artmed.

Clark, M. J. (2018). 'Morbid introspection', unsoundness of mind, and British psychological medicine, c. 1830-c. 1900. In The anatomy of madness (pp. 71101). Routledge. 
Brown, S. M., Rienks, S., McCrae, J. S., \& Watamura, S. E. (2019). The co-occurrence of adverse childhood experiences among children investigated for child maltreatment: A latent class analysis. Child abuse \& neglect, 87, 18-27.

BRASIL. (1990). Presidência da República. Casa Civil. Lei 8.069, de 13 de julho de 1990. Dispõe sobre o Estatuto da Criança e do Adolescente e dá outras providências. 1990. http://www.planalto.gov.br/ccivil_03/Leis/L8069.htm.

Da Silva, J. V., \& Roncalli, A. G. (2018). Prevalence of sexual violence in Brazil: associated individual and contextual factors. International journal of public health, 63(8), 933-944.

Debowska, A., Willmott, D., Boduszek, D., \& Jones, A. D. (2017). What do we know about child abuse and neglect patterns of co-occurrence? A systematic review of profiling studies and recommendations for future research. Child Abuse \& Neglect, 70, 100-111.

Elmore, A. L., \& Crouch, E. (2020). The association of adverse childhood experiences with anxiety and depression for children and youth, 8 to 17 years of age. Academic pediatrics, 20(5), 600-608.

Equestri, A. (2019). 'This cold night will turn us all to fools and madmen': Feste, Lear's Fool and the border between 'idiocy'and mental illness. Cahiers Élisabéthains, 99(1), 23-32.

Facchinetti, C., \& Jacó-Vilela, A. M. (2019). Psychology in Brazilian Assistance to the Insane: first half of the 20th century. Universitas Psychologica, 18(5), $1-15$.

Flick, U. (2008). Uma introdução à pesquisa qualitativa. 3. ed. Porto Alegre: Bookman.

Garlen, J. C., Chang-Kredl, S., Farley, L., \& Sonu, D. (2020). Childhood innocence and experience: Memory, discourse and practice. Children \& Society.

Ghandour, R. M., Sherman, L. J., Vladutiu, C. J., Ali, M. M., Lynch, S. E., Bitsko, R. H., \& Blumberg, S. J. (2019). Prevalence and treatment of depression, anxiety, and conduct problems in US children. The Journal of pediatrics, 206, 256-267.

Hébert, M., Langevin, R., \& Oussaïd, E. (2018). Trauma infantil cumulativo, regulação emocional, dissociação e problemas de comportamento em vítimas de abuso sexual em idade escolar. Jornal de transtornos afetivos , 225 , 306-312.

Kaufman, K. R., Petkova, E., Bhui, K. S., \& Schulze, T. G. (2020). A global needs assessment in times of a global crisis: world psychiatry response to the COVID-19 pandemic. BJPsych open, 6(3).

Kaneko, J. (2021). Ethics in Education for Sustainable Finance: Challenges Toward Long-Termism in Japan and Europe. Handbook on Ethics in Finance, 247268.

Keddie, A. (2018). Adult education: An ideology of individualism. In Adult education for a change (pp. 45-64). Routledge.

Kizilhan, J. I., Steger, F., \& Noll-Hussong, M. (2020). Shame, dissociative seizures and their correlation among traumatised female Yazidi with experience of sexual violence. The British Journal of Psychiatry, 216(3), 138-143.

Kowalczyk, M. H. (2019). Diversified forms of therapeutic and social rehabilitation actions applied to male and female perpetrators of sexual abuse against children. Resocjalizacja Polska, 18(2), 49-63.

Letourneau, EJ, Brown, DS, Fang, X., Hassan, A., \& Mercy, JA (2018). O fardo econômico do abuso sexual infantil nos Estados Unidos. Abuso e negligência infantil , $79,413-422$.

Meyer, M. (2021). Mental Health as Public Health in Rio de Janeiro, Brazil. In Oxford Research Encyclopedia of Latin American History.

Miragoli, S., Camisasca, E., \& Di Blasio, P. (2017). Narrative fragmentation in child sexual abuse: The role of age and post-traumatic stress disorder. Child Abuse \& Neglect, 73, 106-114.

Monteiro, A. C. D. S., Fernandes, A. T. R. S., Oliveira, A. B. M. D., Peixoto, I. V. P., \& Pamplona, M. C. D. C. A. (2018). Mothers' perspective on violence against children: constructing meanings. Revista brasileira de enfermagem,

Nascimento, I. R., Santana, J. P., \& Marques, D. J. C. (2021). Protection of Children and Adolescents from Sexual Violence in Brazil: Legislation and Experiences in a Specialized Police. International Journal of Social Science and Humanity, 11(2).

Pereda, N., \& Díaz-Faes, D. A. (2020). Family violence against children in the wake of COVID-19 pandemic: a review of current perspectives and risk factors. Child and adolescent psychiatry and mental health, 14(1), 1-7.

Paul, O. (2019). Perceptions of family relationships and post-traumatic stress symptoms of children exposed to domestic violence. Journal of family violence, 34(4), 331-343.

Ribeiro, C. H. P., et al. (2018). Transtorno de estresse pós-traumático em vítimas de abuso sexual na infância. Perspectivas Online: Humanas \& Sociais Aplicadas, 8(22).2018.

Russell, D., Higgins, D., \& Posso, A. (2020). Prevenção do abuso sexual infantil: uma revisão sistemática das intervenções e sua eficácia nos países em desenvolvimento. Abuso e negligência infantil , $102,104395$.

Skelton, A. (2019). A hiding to something: Reduction of violence against children in South Africa's justice system. In Violence Against Children in the Criminal Justice System (pp. 145-163). Routledge.

Schlag, F., Allegrini, A. G., Buitelaar, J., Verhoef, E., van Donkelaar, M., Plomin, R., ... \& St Pourcain, B. (2021). Polygenic risk for psychiatric disorder reveals distinct association profiles across social behaviour in the general population. medRxiv. 
Research, Society and Development, v. 10, n. 14, e556101422680, 2021

(CC BY 4.0) | ISSN 2525-3409 | DOI: http://dx.doi.org/10.33448/rsd-v10i14.22680

Sibrava, N. J., Bjornsson, A. S., Pérez Benítez, A. C. I., Moitra, E., Weisberg, R. B., \& Keller, M. B. (2019). Posttraumatic stress disorder in African American and Latinx adults: Clinical course and the role of racial and ethnic discrimination. American Psychologist, 74(1), 101.

Soares, Sandro Vieira; Picolli, Icaro Roberto Azevedo; Casagrande, Jacir Leonir. (2018). Pesquisa bibliográfica, pesquisa bibliométrica, artigo de revisão e ensaio teórico em administração e contabilidade. Administração: ensino e pesquisa, v. 19, n. 2, p. 308-339.

Sullivan, P. F., \& Geschwind, D. H. (2019). Defining the genetic, genomic, cellular, and diagnostic architectures of psychiatric disorders. Cell, 177(1), 162183.

Walker-Descartes, I., Hopgood, G., Condado, L. V., \& Legano, L. (2021). Sexual violence against children. Pediatric Clinics, 68(2), 427-436.

Ward, C. L., Artz, L., Leoschut, L., Kassanjee, R., \& Burton, P. (2018). Sexual violence against children in South Africa: a nationally representative crosssectional study of prevalence and correlates. The Lancet Global Health, 6(4), e460-e468.

Wang, C., Tang, J., \& Liu, T. (2020). The sexual abuse and neglect of "left-behind" children in rural China. Journal of child sexual abuse, 29(5), 586-605.

Williams, N. J., \& Beidas, R. S. (2019). Annual research review: The state of implementation science in child psychology and psychiatry: A review and suggestions to advance the field. Journal of Child Psychology and Psychiatry, 60(4), 430-450. 